\title{
Medidas ecocardiográficas de cavalos de tração da região metropolitana de Curitiba-PR
}

\section{Echocardiographic measurement of cart horses in the metropolitan region of Curitiba-PR}

\author{
Amália Turner Giannico ${ }^{1 *}$; Peterson Triches Dornbusch ${ }^{2}$; Fabiano Montiani- \\ Ferreira $^{2}$; Ivan Roque Barros Filho ${ }^{2}$; Ivan Deconto ${ }^{2}$; Simone Tostes Oliveira ${ }^{2}$
}

\begin{abstract}
Resumo
Para manter o desempenho dos cavalos durante o exercício físico o coração se adapta às variações das atividades metabólicas, o que resulta em adaptações do músculo cardíaco. Desta maneira, o uso da ecocardiografia permite avaliar possíveis adaptações cardíacas e falhas no bombeamento sanguíneo. Pouco se sabe sobre alterações ecocardiográficas em cavalos de tração de materiais recicláveis, que puxam carroças, cujas condições gerais de saúde e manejo quase sempre são inadequadas para o tipo de exercício físico ao qual são submetidos. Objetivou-se estabelecer valores ecocardiográficos para cavalos de carroceiros. Dezenove cavalos sem raça definida foram submetidos ao exame ecocardiográfico, e valores da distância do ponto E (maior abertura da valva mitral) ao septo interventricular; diâmetro da cavidade do ventrículo esquerdo; espessura do septo interventricular e espessura da parede livre do ventrículo esquerdo durante a sístole e diástole; fração de encurtamento e ejeção foram obtidos pelo modo-M. No modo bidimensional foram avaliados o diâmetro da valva aórtica e átrio esquerdo, além de sua relação. Foram avaliadas as velocidades do fluxo de sangue na artéria pulmonar e aorta e valva mitral por meio do Doppler pulsado e/ou contínuo, além de detectar possíveis refluxos por meio do Doppler colorido. Neste estudo ficam estabelecidos os valores médios dos parâmetros ecocardiográficos para os 19 cavalos estudados que fazem trabalho de tração puxando carroças. Não houve valores significativos que constatem remodelamento cardíaco inadequado, disfunção ventricular esquerda com consequente perda de desempenho no exercício ou doenças que prejudiquem o rendimento do animal no trabalho. Palavras-chave: Cardiologia eqüina, ecocardiografia, carroceiro, tração animal
\end{abstract}

\begin{abstract}
In order to maintain the horses' performance during the exercise, their hearts adapt to the variations on metabolic activity, resulting in muscular adaptation. Thus, echocardiography allows to assess on both eventual adaptations of heart tissue and impairment of the ability to pump blood. Little is yet known about echocardiography in cart horses, whose general conditions of health and treatment are often inappropriate to the type of exercise they are submitted to. The goal of this study was to establish echocardiographic numbers for cart horses. Nineteen mongrel horses were submitted for echocardiographic examination, and values of the distance from E point (maximum opening mitral valve) to the interventricular septum; diameter of left ventricle cavity, interventricular septum thickness and thickness of left ventricle free wall during systole and diastole, fractional shortening and ejection fraction were obtained by M-mode. In the 2D images, diameter of the aortic valve and left atrium were evaluated, and their relationship. The velocities of the blood flow were evaluated in aorta and pulmonary artery and the mitral valve by

1 Discente, Universidade Federal do Paraná, UFPR, Curitiba, PR. E-mail: amaliaturner@uol.com.br

2 Profs., UFPR, Curitiba, PR. E-mail: petriches@ufpr.br; montiani@ufpr.br; ivanbarf@ufpr.br; deconto@ufpr.br; simonetostes@
\end{abstract} ufpr.br

* Autor para correspondência 
means of Doppler, and possible insufficiency was detected through the color Doppler. Mean values for echocardiographic parameters were established considering 19 traction horses that worked pulling cart loads. There were no significant values that indicated either an inappropriate cardiac remodeling, a left ventricular dysfunction - with consequent decrease in exercises performance - or diseases that affect the animal's performance at work.

Key words: Equine cardiology, echocardiography, cartwright, animal traction

\section{Introdução}

Para manter o desempenho dos cavalos durante o exercício físico é necessário que o coração seja capaz de bombear sangue na quantidade suficiente para atender as exigências destes animais. Para suprir a nova demanda, o coração se adapta às variações e aumentos das atividades metabólicas, resultando em adaptações bioquímicas, elétricas, morfológicas e mecânicas do músculo cardíaco que, em conjunto, proporcionam uma melhor funcionalidade (BELLO et al., 2011b). Assim, há redução do estresse sobre as paredes ventriculares e, ao mesmo tempo, maior demanda de suprimento sanguíneo. Estas adaptações observadas no coração em consequência dos exercícios vêm sendo descritas na medicina pelo termo "coração atleta" (PELLICIA; MARON, 1997).

O exame ecocardiográfico (ECO) permite uma investigação morfológica e funcional das estruturas cardíacas. As mensurações obtidas por este método diagnóstico determinam os índices da função ventricular, os quais avaliam possíveis falhas no bombeamento sanguíneo (LIGHTOWLER; MERCADO; PAJOT, 1996). Índices cardíacos de cavalos de corrida (YOUNG, 1999) e de cavalos de enduro (MICHIMA et al., 2004), avaliados por meio do ECO, sofreram alterações após um período de treinamento, sugerindo hipertrofia cardíaca excêntrica como resposta adaptativa do coração frente ao exercício. Os cavalos de carroceiros percorrem longas distâncias por períodos prolongados, da mesma forma que cavalos de enduro. As adaptações do organismo em resposta ao exercício são fisiológicas, contudo, estudos realizados nos seres humanos (WELSH et al., 2005; NEILAN et al., 2006) que executam exercício por longos períodos sugeriram evidências ecocardiográficas de disfunção cardíaca, além de mostrarem que esse risco é maior em atletas com baixo nível de treinamento.

Apesar do conhecimento de vários estudos eletrocardiográficos e ecocardiográficos realizados em cavalos submetidos a esforços físicos, tanto de força quanto de resistência (LONG, 1992; YOUNG, 1999; MICHIMA et al., 2004; ZUCCA et al., 2008), pouco se sabe sobre as alterações cardíacas em cavalos de tração, que puxam carroças, cujas condições gerais de saúde e manejo quase sempre são inadequadas para o tipo de exercício físico ao qual são submetidos. Desta forma, objetivou-se estabelecer valores de índices ecocardiográficos para cavalos carroceiros utilizados para tração na região metropolitana de Curitiba-PR.

\section{Material e Métodos}

Um total de 19 cavalos (10 machos e nove fêmeas), sem raça definida, com idade média de 9,0 $\pm 4,7$ anos foi utilizado neste estudo. Estes animais apresentavam peso médio de $362,5 \pm 66,1 \mathrm{~kg}$ (mínimo de $255 \mathrm{~kg}$; máximo de $480 \mathrm{~kg}$; mediana de $380 \mathrm{~kg}$ ). O escore corporal médio foi 2 (escala de $1 \mathrm{a}$ 5). Os cavalos eram utilizados para puxar carroças, transportando principalmente material reciclável, fretes e mudanças. A jornada de trabalho variava de 8 a 13 horas diárias, sendo em alguns momentos o trabalho contínuo e sem pausas e outros com pausas de poucos minutos a duas horas entre uma atividade e outra. Os animais foram atendidos no Hospital Veterinário da Universidade Federal do Paraná (HV-UFPR) por meio do projeto "Carroceiros", que promove atendimento clínico sem custos para os cavalos de carroceiros da cidade de Curitiba e 
região metropolitana - PR. Estes animais em geral chegavam ao HV-UFPR com problemas ortopédicos e feridas, e durante a avaliação, eram submetidos ao ECG e ECO.

O ECO foi realizado com o aparelho de ultrassom My Lab 30 Vet, (The Esaote Group, Genova, Italy) com transdutor setorial e frequência de $2 \mathrm{MHz}$. Foi realizada tricotomia do tórax no lado direito e esquerdo na região do quarto e quinto espaços intercostais, dorsal ao olécrano. Os animais foram mantidos em estação em tronco de contenção durante o exame. Foram utilizados no ECO o modo 2D, modo-M, Doppler colorido, pulsado e contínuo.

Realizou-se o ECO através dos cortes descritos por Patteson et al. (1995) e Schwarzwald (2004). $\mathrm{Na}$ abordagem direita foram realizadas imagens pelo corte paraesternal longitudinal direito da via de saída do ventrículo esquerdo, paraesternal transversal direito do ventrículo esquerdo, da valva mitral, da base cardíaca no nível da aorta e átrio esquerdo e no nível da artéria pulmonar. Nestas imagens foram adquiridos, através do modo-M, os valores da distância do ponto E (maior abertura da valva mitral) ao septo interventricular (E-septo), diâmetro da cavidade do ventrículo esquerdo (DVE), espessura do septo interventricular (SIV) e espessura da parede livre do ventrículo esquerdo (PLVE) durante a sístole e diástole e, a partir destes valores, a fração de encurtamento (FS\%) e fração de ejeção $(\mathrm{FE} \%)$ foram obtidas. No modo $2 \mathrm{D}$ foram obtidos o diâmetro da valva aórtica (DAo), diâmetro do átrio esquerdo (DAE) e consequente relação átrio esquerdo/aorta (AE/Ao). Avaliou-se a velocidade do fluxo de sangue na artéria pulmonar através do Doppler pulsado e/ou contínuo, além de detectar refluxos pela valva pulmonar (VP) através do Doppler colorido.

O volume sistólico final do ventrículo esquerdo (VSF) e volume diastólico final do ventrículo esquerdo (VDF) foram determinados pela fórmula modificada de Teicholz (TEICHOLZ; KREULEN; HERMAN, 1976). Após, o volume de ejeção (VEj) foi obtido pela diferença entre VDF e VSF e o débito cardíaco (DC) foi obtido multiplicando-se o VEj pela frequência cardíaca (FC) e dividindo-se por 1000 para obter o valor em $\mathrm{L} / \mathrm{min}$.

O exame foi continuado pela janela paraesternal esquerda. Por meio de Doppler pulsado e/ou contínuo no corte paraesternal apical esquerdo cinco câmaras quantificou-se a velocidade do fluxo sanguíneo na valva aórtica. Quando não era possível a obtenção do alinhamento adequado, a correção do ângulo foi aplicada pelo programa do aparelho de ultrassom. Foi utilizado Doppler colorido nas valvas aórtica, mitral e tricúspide para detecção de refluxo sanguíneo.

O teste de Shapiro-Wilk foi utilizado para demonstrar a distribuição dos erros amostrais. Após, foram realizadas análises estatísticas descritivas e inferenciais paramétricas das variáveis quantitativas utilizando o programa StatView (SAS, Cary, NC, $E U A$ ), apresentando os resultados sob a forma de média, desvio padrão e intervalo de confiança. Para comparação dos dados ecocardiográficos entre os sexos foi empregado o teste- $t$ não-pareado. Valores de $P<0,05$ foram considerados significativos.

\section{Resultados}

Os valores das médias com os desvios padrão e intervalo de confiança dos índices ecocardiográficos dos cavalos puxadores de carroça estão expressos na Tabela 1. As imagens ecocardiográficas referentes a estes parâmetros podem ser observadas nas Figuras 1 e 2 .

De acordo com o sexo, cinco dos parâmetros avaliados apresentaram diferença estatística. A FC de fêmeas foi maior que a de machos $(P=0,0077)$. O DVEd $(P=0,0047)$ e o DVEs $(P=0,006)$ foram maiores nos machos do que nas fêmeas. A FS\% $(P=0,032)$ e a $\mathrm{FE} \%(P=0,0271)$ foram maiores nas fêmeas do que nos machos. 
Tabela 1. Média com desvio padrão e intervalo de confiança dos parâmetros ecocardiográficos em 19 cavalos puxadores de carroça da região metropolitana de Curitiba-PR.

\begin{tabular}{lcc}
\hline & Média \pm DP* & IC** \\
\hline DAE $(\mathrm{cm})$ & $7,59 \pm 0,88$ & $5,87-9,31$ \\
DAo $(\mathrm{cm})$ & $6,35 \pm 0,74$ & $4,90-7,80$ \\
AE/Ao & $1,19 \pm 0,11$ & $0,97-1,41$ \\
SIVd (cm) & $2,23 \pm 0,39$ & $1,47-2,99$ \\
DVEd (cm) & $8,62 \pm 1,40$ & $5,88-11,36$ \\
PLVEd (cm) & $2,51 \pm 0,41$ & $1,71-3,31$ \\
SIVs (cm) & $3,61 \pm 0,57$ & $2,49-4,73$ \\
DVEs (cm) & $5,44 \pm 1,32$ & $2,85-8,03$ \\
PLVEs (cm) & $3,15 \pm 0,44$ & $2,29-4,01$ \\
FS\% (\%) & $37,47 \pm 6,97$ & $23,81-51,13$ \\
FE\% (\%) & $64,63 \pm 9,48$ & $46,05-83,21$ \\
E-septo (cm) & $1,04 \pm 0,73$ & $0-2,47$ \\
VEj (mL) & $265,54 \pm 83,31$ & $102,25-428,83$ \\
DC (L/min) & $10,59 \pm 2,94$ & $4,83-16,35$ \\
\hline FC (bpm) & $40,47 \pm 5,26$ & $30,16-50,78$ \\
\hline
\end{tabular}

*DP - Desvio padrão

** - Intervalo de confiança

Diâmetro do átrio esquerdo (DAE); diâmetro da aorta (DAo); relação entre átrio esquerdo e aorta (AE/Ao); espessura do septo interventricular na sístole (SIVs); diâmetro do ventrículo esquerdo na sístole (DIVEs); espessura da parede livre do ventrículo esquerdo na sístole (PLVEs); espessura do septo interventricular na diástole (SIVd); diâmetro do ventrículo esquerdo na diástole (DVEd); espessura da parede livre do ventrículo esquerdo na diástole (PLVEd); fração de encurtamento (FS\%); fração de ejeção (FE\%); distância do ponto E ao septo interventricular (E-septo); volume de ejeção (VEj); débito cardíaco (DC); frequência cardíaca (FC).

Fonte: Elaboração dos autores.

Figura 1. Imagem ecocardiográfica de uma égua sem raça definida de seis anos. Corte paraesternal transversal direito da base cardíaca no nível da aorta e átrio esquerdo, no quarto espaço intercostal, em momento de diástole. Transdutor setorial. Modo bidimensional: $2 \mathrm{MHz}$, profundidade $27 \mathrm{~cm}$. Observa-se átrio esquerdo (AE) e artéria aorta (Ao), com seus folhetos valvares fechados. Foi realizada medida do diâmetro dessas estruturas e a relação entre elas. AE - 68,4 $\mathrm{mm}$; Ao - 62,0 mm; AE/Ao - 1,1.

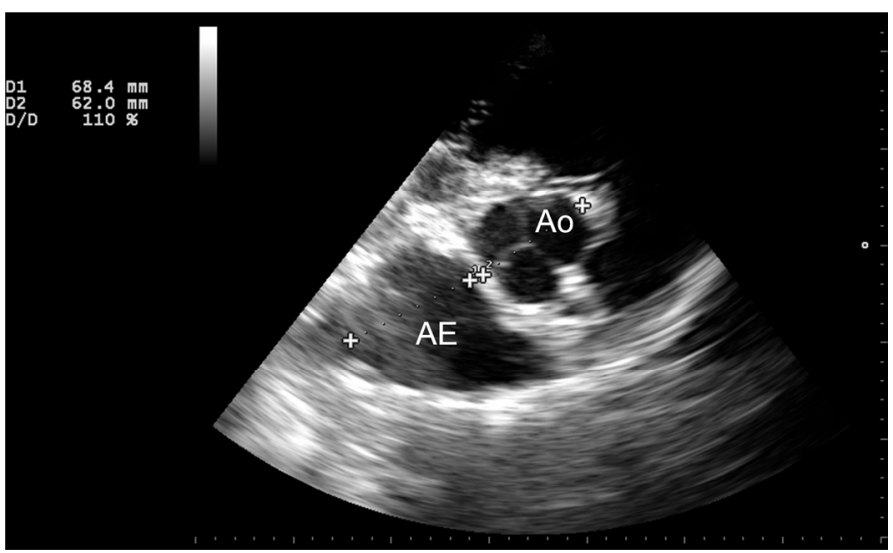

Fonte: Elaboração dos autores. 
Figura 2. Imagem ecocardiográfica de modo-M obtida a partir de imagem de modo-B de corte transversal de ventrículo esquerdo, ilustrando posicionamento dos compassos para mensuração das diversas estruturas, de um cavalo sem raça definida de sete anos. Momento de diástole (seta grossa). Momento de sístole (seta fina). Corte paraesternal transversal direito do ventrículo esquerdo, no quarto espaço intercostal. Transdutor setorial. Modo bidimensional: 2 $\mathrm{MHz}$, profundidade $25 \mathrm{~cm}$. Valores encontrados: espessura do septo interventricular na diástole (SIVd) - 24,5 mm; diâmetro do ventrículo esquerdo na diástole (DVEd) - 90,6 mm; espessura da parede livre do ventrículo esquerdo na diástole (PLVEd) - 28,8 mm; espessura do septo interventricular na sístole (SIVs) - 35,2 mm; diâmetro do ventrículo esquerdo na sístole (DVEs) - 62,9 mm; espessura da parede livre do ventrículo esquerdo na sístole (PLVEs) - 36,2 $\mathrm{mm}$; fração de encurtamento (FS\%) - 31\%; fração de ejeção (FE\%) - 56\%.

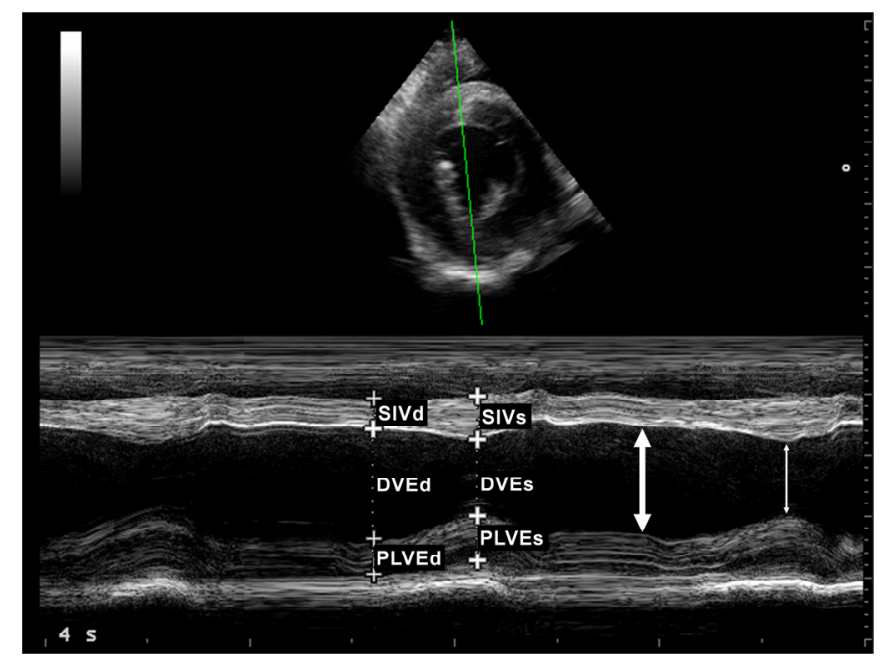

Fonte: Elaboração dos autores.

Na avaliação com Doppler colorido, regurgitação discreta foi encontrada em sete animais (36,85\% dos cavalos avaliados), sendo que três deles apresentavam regurgitação em valva semilunar pulmonar (Figura 3), dois em valva semilunar aórtica, um em valva mitral e valva semilunar aórtica, e um em valva semilunar aórtica e pulmonar.
Foi encontrado como valor médio $1,01 \pm 0,21$ $\mathrm{m} / \mathrm{s}$ para o fluxo sanguíneo aórtico (Figura 4) e $0,60 \pm 0,11 \mathrm{~m} / \mathrm{s}$ para o fluxo sanguíneo pela artéria pulmonar. $\mathrm{O}$ gradiente de pressão teve média de $4,19 \pm 1,9 \mathrm{mmHg}$ na valva aórtica e $1,48 \pm 0,6$ $\mathrm{mmHg}$ na valva pulmonar. 
Figura 3. Imagem ecocardiográfica de um cavalo sem raça definida de seis anos. Corte paraesternal longitudinal direito da via de saída do ventrículo direito, no quarto espaço intercostal, em momento de diástole. Transdutor setorial. Modo bidimensional: $2 \mathrm{MHz}$, profundidade $23 \mathrm{~cm}$. Doppler colorido: 1,6 MHz, PRF 4,2 kHz, ganho de 40\%. Observa-se regurgitação discreta (jato em vermelho, seta) de valva pulmonar. Artéria aorta (Ao); artéria pulmonar (Ap); ventrículo direito (VD).

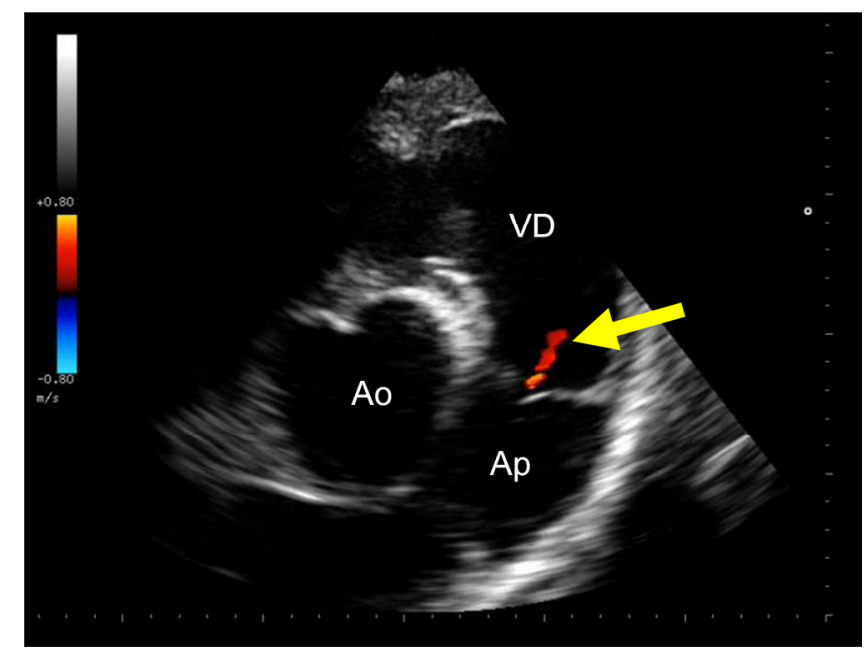

Fonte: Elaboração dos autores.

Figura 4. Gráfico de fluxo aórtico obtido por Doppler contínuo, exemplificando mensuração de velocidade (111,0 $\mathrm{cm} / \mathrm{s})$ e gradiente de pressão $(4,9 \mathrm{mmHg})$ de uma égua sem raça definida de dez anos. Corte paraesternal apical esquerdo cinco câmaras, no quinto espaço intercostal. Transdutor setorial. Modo bidimensional: $2 \mathrm{MHz}$, profundidade $19 \mathrm{~cm}$. Artéria aorta (Ao); átrio esquerdo (AE); ventrículo esquerdo (VE); ventrículo direito (VD).

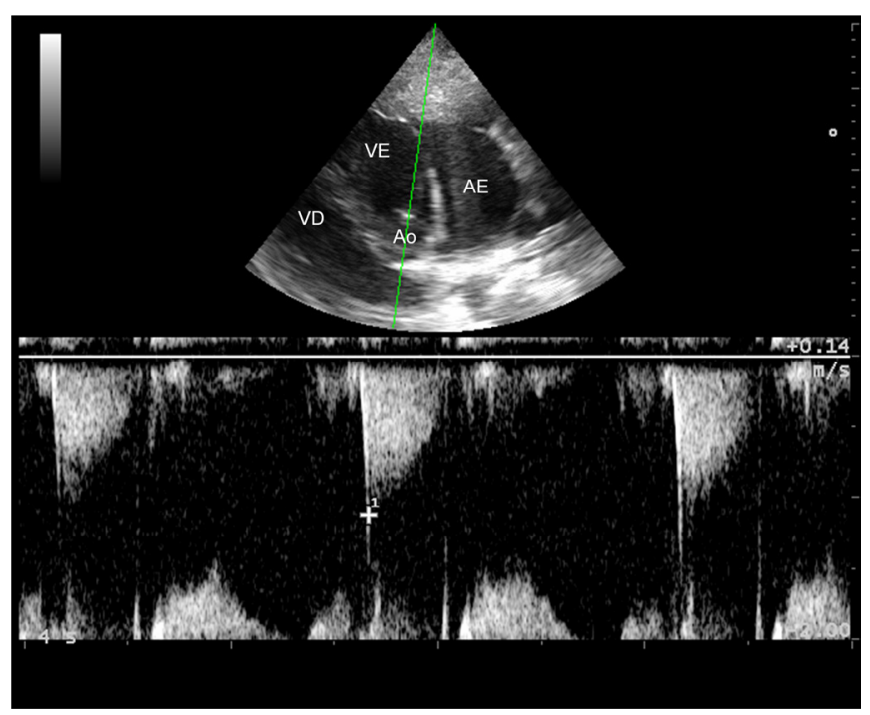

Fonte: Elaboração dos autores. 


\section{Discussão}

A FC média encontrada neste estudo foi de 40,47 \pm 5,26 bpm, valor próximo ao de Diniz (2006) que avaliou cavalos de salto e Dumont et al. (2010) que avaliaram cavalos da raça Puro Sangue Árabe de enduro, e obtiveram como FC média 40,20 bpm e 35,85 bpm, respectivamente. Houve diferença em relação a um estudo que verificou variação de 49 a 51 bpm para cavalos Mangalarga e mestiços submetidos à prova de enduro (FERNANDES, 1994). Esta menor FC observada neste estudo pode sugerir que tal característica ocorreu em função da adaptação cardíaca e vascular em decorrência ao esforço físico rotineiro.

A FC encontrada em éguas foi maior do que em cavalos, de forma semelhante ao encontrado por Fernandes et al. (2004); Diniz (2006) e Diniz et al. (2008). Não há pesquisas na cardiologia equina que expliquem estas diferenças entre sexos. Talvez fatores hormonais possam ser a causa, como mostram pesquisas na medicina humana (HUIKURI et al., 1996; LIU; KUO; YANG, 2003).

$\mathrm{Na}$ avaliação ecocardiográfica dos animais, observou-se que o tamanho do $\mathrm{AE}$ e da Ao foram próximos, sendo sua relação de 1,19. Esta relação difere de Bonomo et al. (2011), que utilizaram o mesmo método de mensuração do $\mathrm{AE}$ deste presente trabalho. Estes autores verificaram relação AE/Ao de 1,5 em cavalos atletas da raça Quarto de Milha que realizavam provas de baliza e tambor. Na literatura, há divergências entre os métodos de mensuração do AE entre os trabalhos publicados, sendo diversos os métodos conhecidos para estimar o tamanho do AE. Esta mensuração pode ser realizada utilizando o modo-M ou o modo-B, analisando a relação do diâmetro do AE com o peso corporal ou área de superfície corporal, ou relacionando o diâmetro do $\mathrm{AE}$ com o diâmetro da aorta. Boon (2011) sugeriu outras duas medidas para a mensuração do AE, além da avaliação por meio do corte paraesternal transversal direito, onde o tamanho do AE é relacionado com a aorta: por meio do corte paraesternal direito quatro câmaras, onde o AE pode ser mensurado no sentido anteroposterior e basilar-apical. É provável que cada mensuração tenha um significado clínico, mas pouco se sabe sobre a interpretação destes dados tanto em cavalos como em outras espécies. Desta maneira, o tamanho do átrio esquerdo e a relação $\mathrm{AE} / \mathrm{Ao}$ devem ser interpretados com cautela, pois podem diferir quando comparados a estudos com cavalos que utilizaram métodos de mensuração diferentes, como a mensuração da totalidade da câmara atrial esquerda. Em cavalos Puro Sangue e Quarto de Milha, que são animais com maior peso que os cavalos puxadores de carroça deste estudo, o valor para o diâmetro da Ao é de aproximadamente $7 \mathrm{~cm}$ (PATTESON et al., 1995; BONOMO et al., 2011). Os cavalos estudados por nós são menores que os cavalos atletas de outras raças estudadas e, desta maneira, já era esperado menor diâmetro da aorta, que teve média de $6,35 \pm 0,74 \mathrm{~cm}$. Já comparando este diâmetro com os valores encontrados em pôneis entre 274 e $469 \mathrm{~kg}$, verifica-se um valor mais próximo, onde o encontrado nestes animais é de 5,9 $\pm 1,0 \mathrm{~cm}$ (SLATER; HERRTAGE, 1995). O AE teve valor semelhante à Ao, com média de 7,59 $\pm 0,88 \mathrm{~cm}$. Patteson et al. (1995) encontraram valores maiores $(12,82 \pm 0.78 \mathrm{~cm})$ para animais Puro Sangue cujo peso é superior aos cavalos puxadores de carroça. Não há muitos trabalhos sobre o tamanho atrial e aórtico e sua relação na literatura consultada, mas por meio deste trabalho realizado, acredita-se que os tamanhos do AE e da Ao devam ser próximos, e sua relação deva ser semelhante ao padrão para outras espécies, que tem relação próxima a 1:1 (BOON, 2011).

Os valores encontrados de espessura de septo interventricular, diâmetro interno de ventrículo esquerdo e parede livre do ventrículo esquerdo, tanto na sístole como na diástole, foram menores que os valores observados por Bonomo et al. (2011) ao estudar cavalos Quarto de Milha que realizavam provas de baliza e tambor. Estes esportes têm duração rápida e basicamente utiliza-se no exercício 
físico a via anaeróbica, levando principalmente a um aumento da massa miocárdica. Também se observam valores menores para estes parâmetros quando comparados com cavalos Puro Sangue (MARR; PATTESON, 2010), Como os animais estudados apresentaram peso inferior ao destes cavalos, estes valores menores já eram esperados, como discutido anteriormente. Comparando-se os valores dos cavalos do presente estudo aos citados na literatura para animais com peso aproximado, como os pôneis (peso entre 274 e $469 \mathrm{~kg}$ ) (SLATER; HERRTAGE, 1995), observou-se que os valores encontrados nos pôneis apresentaram-se ligeiramente inferiores ao dos cavalos puxadores de carroça. Os cavalos de carroceiros são considerados animais pequenos, quando comparados aos cavalos de esporte, porém apresentam porte semelhante aos cavalos da raça Crioula (KURTZ-FILHO; LÖF, 2007). Não há trabalhos na literatura com animais da desta raça, a que seria a mais adequada para comparação devido ao peso semelhante.

Houve diferença estatística para os valores do DVEd e do DVEs quando relacionado os sexos, observando-se valores maiores nos cavalos do que nas éguas. Esta diferença não pode ser explicada pelo peso, já que não houve diferença estatística do peso entre machos e fêmeas.

A distância do ponto E-septo foi similar à encontrada por Bonomo et al. (2011), que observou média de $1,4 \pm 0,38 \mathrm{~cm}$, e maior do que o observado por Zucca et al. (2008), que teve como média 0,59 $\pm 0,21 \mathrm{~cm}$. Este parâmetro deve ser cuidadosamente interpretado, já que seu método de mensuração é sujeito a erros em consequência de um adequado posicionamento do transdutor, além de haver variações secundárias às diferentes aptidões atléticas e fase do remodelamento cardíaco. Como exemplo, se há hipertrofia concêntrica do VE secundária ao exercício, a distância E-septo pode diminuir. O E-septo é um indicador de enchimento e função do ventrículo esquerdo (BOON, 2011). Doenças cardíacas podem aumentar, diminuir ou não afetar este parâmetro. Apesar disso, E-septo tem forte correlação negativa com a fração de ejeção, ou seja, um aumento do seu valor denota diminuição da fração de ejeção (KIRBERGER, 1991). Desta maneira, sugere-se que, quando novas pesquisas com ecocardiografia em cavalos forem realizadas, este parâmetro deva estar presente, uma vez que poucos trabalhos realizaram esta medição.

A FS\% foi semelhante ao encontrado em cavalos da raça Puro Sangue Inglês (PATTESON et al., 1995); maior que o encontrado em Standardbreds (ZUCCA et al., 2008) e em animais da raça Puro Sangue Árabe que praticam enduro (BELLO et al., 2011b); e menor que o encontrado em animais da raça Quarto de Milha que realizam provas de baliza e tambor (BONOMO et al., 2011). A FE\% foi menor do que o encontrado em animais que praticam polo (BELLO et al., 2011a; BONOMO et al., 2011). Segundo Reef (1990), a FS\% e FE\% podem variar de acordo com a idade, peso e raça do animal, e que, na maioria dos cavalos em repouso com $\mathrm{FC}$ variando de 30 a $40 \mathrm{bpm}$, a FS\% varia de 30 a 40\%. O presente estudo corroborou com Reef (1990), pois os valores da FS\% e FE\% estão entre os valores de referência descritos, mas observouse diferença estatística entre os sexos, onde fêmeas apresentaram maiores valores nestes parâmetros do que machos, porém nenhum motivo aparente para este fato foi identificado.

A FS\% não é uma medida de contratilidade, mas de função e complacência do VE. Quando um valor baixo de FS\% é obtido, este pode ser secundário à baixa pré-carga, alta pós-carga ou contratilidade diminuída. Apesar dos fatores primários serem de difícil diferenciação, as medidas em modo-M são de grande auxílio. O aumento de tamanho diastólico do VE sugere que uma diminuição da pré-carga não é o fator determinante para a baixa $\mathrm{FS} \%$, enquanto a diminuição no tamanho de câmara diastólica do VE é tanto uma indicação de baixa pré-carga, como de alta pós-carga, se as hipertrofias de parede e de septo estiverem presentes (BOON, 2011). Desta maneira, as diferentes observações em relação à $\mathrm{FS} \%$ e $\mathrm{FE} \%$ entre os autores podem estar relacionadas 
ao remodelamento cardíaco secundário ao tipo de exercício físico que os animais estão submetidos em cada estudo. Uma maior força de contração é exigida durante um exercício de alta intensidade como as provas de tambor e de baliza, e o coração deve, neste caso, contrair com mais força e maior intensidade para compensar a rápida demanda, quando comparado com cavalos de provas de enduro. Já quando comparados com cavalos de corrida essa diferença pode ser explicada pelo fato destes animais apresentarem dimensões cardíacas maiores, exigindo proporcionalmente, uma maior força de contração para corresponder à demanda do exercício.

$\mathrm{O}$ VEj e DC foram menores do que o encontrado por Bonomo et al. (2011) e Bello et al. (2011b). O VEj depende do diâmetro da cavidade do ventrículo esquerdo tanto em sístole como em diástole e o DC depende do VEj e da FC. Desta maneira os cavalos terão $\mathrm{VEj}$ e DC diferentes de acordo com o peso, tipo de treinamento e consequente tamanho das câmaras cardíacas.

$\mathrm{Na}$ avaliação com Doppler colorido observouse regurgitação discreta em 36,85\% dos cavalos avaliados, onde o maior número de animais tinha regurgitação na valva pulmonar, seguido pela valva aórtica. Fraipont et al. (2011) observaram em cavalos de enduro regurgitação valvar que variou de leve a moderada, sendo a valva mais afetada a aórtica (41,9\% dos cavalos com regurgitação) seguido pela pulmonar (22,6\% dos cavalos com regurgitação) e mitral (22,6\% dos cavalos com regurgitação). Os mesmos autores avaliaram a diminuição do desempenho de cavalos de enduro em relação à regurgitação valvar. Eles descrevem que este fato não está associado com diminuição do desempenho atlético dos animais. Outro estudo também não encontrou associação negativa entre alta prevalência de regurgitação valvar e desempenho dos cavalos de corrida e enduro (YOUNG; SCOTT, 1998).

Apesar de a ultrassonografia com o uso do Doppler ser considerada limitada na cardiologia equina, devido à dificuldade de alinhar paralelamente o feixe de ultrassom ao fluxo sanguíneo, foram avaliados no presente trabalho os fluxos nas artérias aorta e pulmonar, conseguindo-se, na maioria das vezes, adequado alinhamento entre o fluxo e o cursor do Doppler pulsado e contínuo. Apenas o trabalho de Young e Scott (1998) fornece parâmetros para estes dois fluxos, descrevendo para o fluxo aórtico valor semelhante $(1,03 \mathrm{~m} / \mathrm{s})$ ao observado nos cavalos puxadores de carroça deste estudo. Já em relação ao fluxo na artéria pulmonar, o valor encontrado nos cavalos puxadores de carroça $(0,60 \pm 0,11 \mathrm{~m} / \mathrm{s})$ foi menor do que o valor encontrado por estes mesmos autores $(0,94 \mathrm{~m} / \mathrm{s})$. A habilidade da ecocardiografia Doppler em estimar a velocidade do fluxo sanguíneo de forma nãoinvasiva abriu novas áreas de investigação para a detecção e quantificação de várias lesões cardíacas. Uma de suas utilidades é a complementação da avaliação da função sistólica cardíaca por meio da avaliação dos fluxos das valvas aórtica e pulmonar (PETRUS et al., 2010). Estudos realizados em pessoas (GARDIN et al., 1993; IMAMURA et al., 1993) mostraram que a ecocardiografia Doppler tem a vantagem de refletir, quantitativamente, a função de todo o ventrículo esquerdo, enquanto a fração de encurtamento do ventrículo esquerdo, medida pelo modo unidimensional, reflete o valor da função de uma área específica do coração. Desta maneira, seria interessante que as novas pesquisas com ecocardiografia em cavalos começassem a aferir e quantificar estes parâmetros.

No presente estudo, observaram-se medidas do ventrículo esquerdo levemente maiores do que o observado em pôneis de peso aproximado. Apesar deste achado, os cavalos puxadores de carroça parecem não apresentar remodelamento cardíaco secundário ao exercício que são submetidos diariamente. Para uma adequada associação entre o tipo do exercício que os cavalos puxadores de carroça são submetidos e seu consequente remodelamento cardíaco, causando aumento das câmaras cardíacas, devem ser realizadas outras pesquisas, aferindo- 
se os parâmetros ecocardiográficos antes dos animais serem submetidos ao trabalho. O esperado previamente neste estudo era encontrar nestes animais aumento da câmara ventricular esquerda (hipertrofia excêntrica) e um aumento do volume sistólico como resposta adaptativa do coração ao exercício de resistência. Tanto na medicina humana como na veterinária há trabalhos sobre remodelamento do coração frente aos exercícios físicos. Alguns autores relatam remodelamento, já outros não comprovam alterações na estrutura e função cardíaca. Em estudos feitos na medicina com atletas de remo, ciclismo, natação e provas de triathlon que percorrem longas distâncias, foi observado que o treinamento de resistência realizado por longos períodos (4 a 17 anos) não foi associado com mudanças significativas no tamanho e função do ventrículo esquerdo ou com a ocorrência de sintomas cardiovasculares (PELLICCIA et al., 2010). Em oposição, Benito et al. (2011) ao usar ratos condicionados a correr vigorosamente, verificou neste modelo animal fibrose cardíaca após longo período de treinamento físico, juntamente com alterações na função ventricular e aumento na indução de arritmias, chegando à conclusão de que o treinamento físico vigoroso de resistência pode, a longo prazo, promover remodelamento e produzir um substrato para arritmias cardíacas.

Estudos demonstram que em pessoas e em cavalos de corrida de resistência, o exercício prolongado em dias sucessivos é mais deletério para a função cardíaca do que uma única sessão de exercício extenuante, podendo induzir a fadiga cardíaca. (MCGAVOCK et al., 2002; MIDDELTON et al., 2006; SHAVE et al., 2008). Com base na literatura já existente, aparentemente não houve remodelamento cardíaco, o que sugere que a atividade física a que estes animais são submetidos não é deletéria ao coração. Como discutido anteriormente, são necessários novos estudos com população de cavalos de características semelhantes sem nenhum tipo de atividade física prévia, para melhor conhecimento de alterações cardíacas.

\section{Conclusões}

Neste estudo ficam estabelecidos os valores médios dos parâmetros ecocardiográficos para a população estudada, composta por cavalos que fazem trabalho de tração puxando carroças. Apesar dos animais serem submetidos a condicionamento de várias horas de trabalho, o exame ecocardiográfico não detectou valores anormais que constatem remodelamento inadequado ou disfunção do VE com consequente perda de desempenho no exercício e doenças cardíacas que prejudiquem o rendimento do animal no trabalho.

\section{Referências}

BELlO, C. A. O.; LIMA, E. M. M.; SILVA, C. E. V.; GODOY, R. F.; TEIXEIRA-NETO, A. R. Estabelecimento de índices ecocardiográficos de cavalos de polo. Pesquisa Veterinária Brasileira, Seropédica, v. 31, n. 6, p. 495-498, 2011 a.

BELLO, C. A. O.; VASCONCELOS, C. E. S.; GODOY, R. F.; TEIXEIRA-NETO, A. R.; BORGES, J. R. J.; LIMA, E. M. M. Ecocardiografia de equinos Puro Sangue Árabe após exercício de enduro de diferentes intensidades. Ciência Rural, Santa Maria, v. 41, n. 1, p. 132-136, $2011 \mathrm{~b}$.

BENITO, B.; GAY-JORDI, G.; SERRANO-MOLLAR, A.; GUASCH, E.; SHI, Y.; TARDIF, J.; BRUGADA, J.; NATTEL, S.; MONT, L. Cardiac arrhythmogenic remodeling in a rat model of long-term intensive exercise training. Circulation, Boston, v. 123, n. 1, p. 13-22, 2011.

BONOMO, C. M. M.; MICHIMA, L.E. S.; MIYASHIRO, P.; FERNANDES, W. R. Ecocardiografia quantitativa de equinos atletas da raça Quarto de Milha. ARS Veterinária, Jaboticabal, v. 27, n. 4, p. 220-225, 2011.

BOON, J. A. Evaluation of size, function, and hemodynamics. In: .Veterinaryechocardiography. 2. ed. West Sussex: Wiley-Blackwell, 2011. p. 105-196.

DINIZ, M. P. Perfil eletrocardiográfico de equinos de salto criados em São Paulo. 2006. Dissertação (Mestrado em Medicina Veterinária) - Curso de Pós-Graduação em Medicina Veterinária. Universidade de São Paulo, São Paulo.

DINIZ, M. P.; MUZZI, R. A. L.; MUZZI, L. A. L.; ALVES, G. E. S. Estudo eletrocardiográfico de equinos da raça Mangalarga Marchador. Arquivo Brasileiro de Medicina Veterinária e Zootecnia, Belo Horizonte, v. 60, n. 3, p. 536-542, 2008. 
DUMONT, C. B. S.; LEITE, C. R.; MORAES, J. M.; ALVES, R. O.; GODOY, R. F.; LIMA, E. M. M. Parâmetros eletrocardiográficos de equinos Puro Sangue Árabe submetidos a exercício prolongado de enduro. Ciência Rural, Santa Maria, v. 40, n. 9, p. 1966-1973, 2010.

FERNANDES, W. R.; LARSSON, M. H. M. A.; ALVES, A. L. G.; FANTONI, D. T.; BELLI, C. B. Características eletrocardiográficas em equinos clinicamente normais da raça Puro Sangue Inglês. Arquivo Brasileiro de Medicina Veterinária e Zootecnia, Belo Horizonte, v. 56, n. 2, p. 143-149, 2004.

FERNANDES, W. R. Alterações dos parâmetros do eletrocardiograma e da crase sanguínea em equinos das raças Árabe e Mangalarga, bem como de Mestiços, submetidos à prova de enduro. 1994. Tese (Doutorado em Medicina Veterinária) - Curso de Pós-graduação em Medicina Veterinária. Universidade de São Paulo, São Paulo.

FRAIPONT, A.; VAN ERCK, E.; RAMERY, E.; RICHARD, E.; DENOIX, J. M.; LEKEUX, P.; ART, T. Subclinical diseases underlying poor performance in endurance horses: diagnostic methods and predictive tests. Veterinary Record, London, v. 169, n. 6, p. 154, 2011.

GARDIN, J. M.; ISERI, L. T; ELKAYAM, U.; TOBIS, J.; CHILDS, W.; BURN, C. S.; HENRY, W. L. Evaluation of dilated cardiomyopathy by pulsed Doppler echocardiography. American Heart Journal, Durham, v. 106, n. 5, p. 1057-1065, 1993.

HUIKURI, H. V.; PIKKUJAMSA, S. M.; AIRAKSINEN, K. E. J.; IKÄHEIMO, M. J.; RANTALA, A. O.; KAUMA, H.; LILJA, M.; KESÄNIEMI, Y. A. Sexrelated differences in autonomic modulation of heart rate in middle-aged subjects. Circulation, Boston, v. 94, n. 2, p. $122-125,1996$.

KIRBERGER, R. Mitral valve E point to ventricular septal separation in the dog. Journal of the South African Veterinary Association, Durbanville, v. 62, n. 4, p. 163166, 1991.

IMAMURA, Y.; HIRAI, H.; HARADA, M.; TOKUYAMA, A.; YOSHINUMA, M.; DEGAWA, T.; NISHIZAWA, S.; YABUKI, S.; MACHII, K.; YAMAGUCHI, T. Evaluation of left ventricular systolic function by pulsed Doppler echocardiography in patients with acute myocardial infarction. Journal of Cardiology, Okayama, v. 23, n. 3, p. 231-240, 1993.

KURTZ-FILHO, M.; LÖF, H. K. Biometria de equinos da raça crioula no Brasil. Archives of Veterinary Science, Curitiba, v. 12, n. 1, p. 47-51, 2007.
LIGHTOWLER, C. H.; MERCADO, M. C.; PAJOT, S. M. Valor diagnóstico y pronóstico de los indices ventriculares en las enfermedades cardiacas por fallo de bomba. Pet's, Buenos Aires, v. 12, n. 64, p. 177-181, 1996.

LIU, C. C; KUO, T. B.; YANG, C. C. Effects of estrogen on gender related autonomic differences in humans. American Journal of Physiology - Heart and Circulatory Physiology, Bethesda, v. 285, n. 5, p. 2188-2193, 2003.

LONG, K. J. Two-dimensional and M-mode echocardiography. Equine Veterinary Education, Oxford, v. 4, n. 6, p. 303-310, 1992.

MARR, C. M.; PATTESON, M. Echocardiography. In: MARR, C. M.; BOWEN, I. M. Cardiology of the horse. 2. ed. London: Saunders, 2010. p. 105-126.

MCGAVOCK, J.; WARBURTON, D. E.; TAYLOR, D.; WELSH, R. C.; QUINNEY, H. A.; HAYKOWSKY, M. $\mathrm{J}$. The effects of prolonged strenuous exercise on left ventricular function: a brief review. Heart Lung, New Haven, v. 31, n. 4, p. 279-292, 2002.

MICHIMA, L. E. S.; LATORRE, S. M.; ANDRADE, A. F. C.; FERNANDES, W. R. B-mode and M-mode echocardiography of endurance horses raised in São Paulo State. Brazil. Journal of Equine Veterinary Science, Lexington, v. 24, n. 10, p. 451-457, 2004.

MIDDELTON, N.; SHAVE, R.; GEORGE, K.; WHYTE, G.; HART, E.; ATKINSON, G. Left ventricular function immediately following prolonged exercise: a metaanalysis. Medicine \& Science in Sports \& Exercise, Hagerstown, v. 38, n. 4, p. 681-687, 2006.

NEILAN, T. G.; JANUZZI, J. L.; LEELEWANDROWSKI, E.; TON-NU, T. T.; YOERGER, D. M.; JASSAL, D. S.; LEWANDROWSKI, K. B.; SIEGEL, A. J.; MARSHALL, J. E.; DOUGLAS, P. S.; LAWLOR, D.; PICARD, M. H.; WOOD, M. J. Among nonelite participants in the Boston marathon myocardial injury and ventricular dysfunction related to training levels. Circulation, Boston, v. 114, n. 22, p. 2325-2333, 2006.

PATTESON, M. W.; GIBBS, C.; WOTTONET, P. R.; CRIPPS P. J. Echocardiographic measurements of cardiac dimensions and indices of cardiac function in normal adult thoroughbred horses. Equine Veterinary Journal Supplements, Hobokken, v. 27, n. 19, p. 18-27, 1995.

PELLICCIA, A.; KINOSHITA, N.; PISICCHIO, C.; QUATTRINI，F.; DIPAOLO, F. M.; CIARDO, R.; DI GIACINTO, B.; GUERRA, E.; DE BLASIIS, E.; CASASCO, M.; CULASSO, F.; MARON, B. J. Long- 
term clinical consequences of intense, uninterrupted endurance training in olympic athletes. Journal of the American College of Cardiology, San Diego, v. 55, n. 15, p. 1619-1625, 2010.

PETRUS, L. C.; OLIVEIRA, V. M. C.; PEREIRA, G. G.; LARSSON, M. H. M. A. Avaliação dos fluxos das valvas aórtica e pulmonar com ecocardiografia Doppler pulsátil em cães clinicamente sadios. Pesquisa Veterinária Brasileira, Seropédica, v. 30, n. 7, p. 586-592, 2010.

PELLICIA, A.; MARON, B. J. Outer limits of the athlete's heart: the effect of gender and relevance to the differential diagnosis with primary cardiac diseases. Cardiology Clinics, Amsterdam, v. 15, n. 3, p. 381-396, 1997.

REEF, V. B. Echocardiographic examination in the horse: the basics. Compendium on Continuing Education for the Veterinarian Practicing, Yardley, v. 12, n. 9, p. 1312-1319, 1990.

SCHWARZWALD, C. C. Equine echocardiography. 2004. 8 p. Disponível em: <http://minnie.uab. es/ veteri/21231/Echocardio.pdf $>$. Acesso em: 25 mar. 2012.

SHAVE, R.; GEORGE, K.; WHYTE, G.; HART, E.; MIDDLETON, N. Postexercise changes in left ventricular function: the evidence so far. Medicine \& Science in Sports \& Exercise, Hagerstown, v. 40, n. 8, p. 1393-1399, 2008.
SLATER, J. D.; HERRTAGE, M. E. Echocardiographic measurements of cardiac dimensions in normal ponies and horses. Equine Veterinary Journal, Malden, v. 19, n. 2, p. 28-32, 1995.

TEICHOLZ, L. E.; KREULEN, T.; HERMAN, M. V. Problems in echocardiographic volume determinations: echocardiographic-angiographic correlations in the presence or absence of a synergy. American Journal of Cardiology, Dallas, v. 37, n. 1, p. 7-11, 1976.

WELSH, R. C.; WARBURTON, D. E. R.; HUMEN, D. P.; TAYLOR, D. A.; MCGAVOCK, J.; HAYKOWSKY, M. J. Prolonged strenuous exercise alters the cardiovascular response to dobutamine stimulation in male athletes. Journal of Physiology, Oxford, v. 569, n. 1, p. 325-330, 2005.

YOUNG, L. E. Cardiac responses to training in 2-yearold throughbreds: an echocardiographic study. Equine Veterinary Journal, Malden, v. 30, n. 10, p. 195-199, 1999.

YOUNG, L. E.; SCOTT, G. R. Measurement of cardiac function by transthoracic echocardiography: day to day variability and repeatability in normal thoroughbred horses. Equine Veterinary Journal, Malden, v. 30, n. 2, p. 117-122, 1998.

ZUCCA, E.; FERRUCCI, F.; CROCI, C.; DI FABIO, V.; ZANINELLI, M.; FERRO, E. Echocardiographic measurements of cardiac dimensions in normal Standardbred racehorses. Journal of Veterinary Cardiology, West Lafayette, v. 10, n. 1, p. 45-51, 2008. 